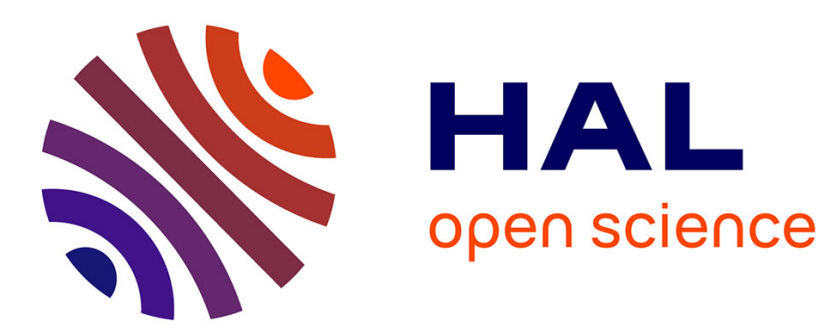

\title{
A note on the ordinal equivalence of power indices in games with coalition structure
}

Sébastien Courtin, Bertrand Tchantcho

\section{To cite this version:}

Sébastien Courtin, Bertrand Tchantcho. A note on the ordinal equivalence of power indices in games with coalition structure. Theory and Decision, 2015, 78 (4), pp.617 - 628. 10.1007/s11238-014-9445-0 . hal-00914910

\section{HAL Id: hal-00914910 https://hal.science/hal-00914910}

Submitted on 6 Dec 2013

HAL is a multi-disciplinary open access archive for the deposit and dissemination of scientific research documents, whether they are published or not. The documents may come from teaching and research institutions in France or abroad, or from public or private research centers.
L'archive ouverte pluridisciplinaire HAL, est destinée au dépôt et à la diffusion de documents scientifiques de niveau recherche, publiés ou non, émanant des établissements d'enseignement et de recherche français ou étrangers, des laboratoires publics ou privés. 


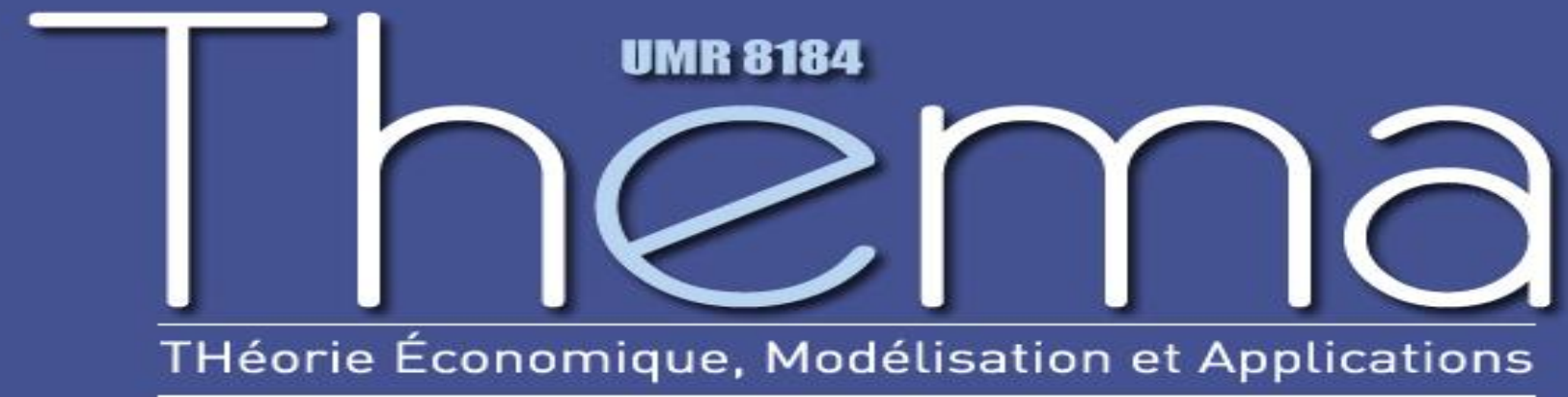

Thema Working Paper $n^{\circ}$ 2013-30

Université de Cergy Pontoise, France

A note on the ordinal equivalence of power indices in games with coalition structure

Sébastien Courtin

Bertrand Tchantcho

May, 2013 


\title{
A note on the ordinal equivalence of power indices in games with coalition structure
}

\author{
Sébastien Courtin* Bertrand Tchantcho ${ }^{\dagger}$
}

May 2013

\begin{abstract}
The desirability relation was introduced by Isbell (1958) to qualitatively compare the a priori influence of voters in a simple game. In this paper, we extend this desirability relation to simple games with coalition structure. In these games, players organize themselves into a priori disjoint coalitions. It appears that the desirability relation defined in this paper is a complete preorder in the class of swap-robust games. We also compare our desirability relation with the preorders induced by the generalizations to games with coalition structure of the Shapley-Shubik and Banzahf-Coleman power indices (Owen, 1977, 1981). It happens that in general they are different even if one considers the subclass of weighed voting games. However, if structural coalitions have equal size then both Owen-Banzhaf and the desirability preordering coincide.
\end{abstract}

Keywords Voting games; Coalition structure; Power indices; Desirability relation

JEL Classification C71, D72

*Corresponding author. University of Cergy-Pontoise, THEMA, UMR CNRS 8184, France. Email: sebastien.courtin@u-cergy.fr

†University of Yaounde I, Advanced Teachers' Training College, Cameroon; and University of Cergy-Pontoise, THEMA, UMR CNRS 8184, France. Email: btchantcho@yahoo.fr 


\section{Introduction}

Power is an important concept in the study of simples games. Power indices are quantitative measures and indicators that characterize the game. In the literature, we find several power indices. The most famous power indices are those of Shapley-Shubik (Shapley and Shubik, 1954) and Banzhaf-Coleman (Banzhaf, 1965; Coleman, 1971). There are many other indices not discussed in this paper (see Andjiga et al. (2003), Laruelle and Valenciano (2008) for a detailed description of power indices).

Because of the multiplicity of notions of power indices in simple games, it seems natural and important that comparisons between them be made. The idea is to base the comparison on their corresponding preorderings. Tomiyama (1987) proved that, for every weighted game, the preorderings induced by the classical Shapley-Shubik $(S S)$ and Banzhaf-Coleman $(B C)$ indices coincide. He calls this property the "ordinal equivalence" of the two indices.

Instead of measuring the players' voting power with an index, the desirability relation (Isbell, 1958; Allingham, 1975; Taylor, 1995) consists in ranking them with respect to how much infuential they are. A given voter who is never needed in any minimal winning coalition may be regarded as not being of any influence at all. On the contrary, if that player is indispensable to every minimal winning coalition, we may think that he is very influential. Most often, it is within these two extreme limits that the majority of voters lie. The ranking of voters with respect to their influence is called the desirability relation. It is a preordering in the set of players.

Diffo Lambo and Moulen (2002) characterize simple games for which the preorderings induced by $S S, B C$ and the desirability preordering coincide. They proved that the three coincide if and only if the simple game is swaprobust (see Section 3). Since any weighted game is swap-robust, hence Diffo Lambo and Moulen (2002) generalize Tomiyama's result.

The problem with the traditional power indices which are based only on the set of winning coalitions, is that they do not take into consideration a priori relations between different players. Indeed in many negotiations some players prefer to cooperate with each other rather than with other players due to the existence of common interests. For example, consider international diplomatic relations, especially those of France and the United States. France belongs to the European Union, which is an a priori coalition of European 
countries, whereas the USA belongs to an a priori coalition with Mexico and Canada (NAFTA). Classical values do not take into consideration this kind of relationship. In order to represent these cooperation situations in a realistic way, some authors (see Aumann and Drèze 1974, Owen 1977 and 1981, among others) introduced games with a priori coalition structure. A coalition structure is a partition of the player set in disjoint coalitions. In such a game it is supposed that players organize themselves to defend their interests into a priori disjoint coalitions (structural coalitions). Owen (1977, 1981) proposed and characterized a modification of $S S$ and $B C$ indices with respect to a coalition structure, the well-known Owen-Shapley $(O S)$ and Owen-Banzhaf $(O B)$ indices. In this case there is a two-level interaction between players. Firstly, coalitions play an external game among themselves, and each one receives a payoff; secondly, in internal games the payoffs of each coalition are distributed amongst their members. Both payoffs, in the external game and in the internal game, are given by the Shapley value or the Banzhaf value.

In this paper, along the line of Tomiyama (1987) and Diffo Lambo and Moulen (2002), we conduct an ordinal comparison of the desirability relation with the preorderings induced in the set of player of a simple game with coalition structure (denoted $S G C S$ ) by $O S$ and $O B$ indices, respectively.

First of all, we extend the desirability relation to $S G C S$. We show that this relation generalizes the desirability relation for simple games. Moreover, we extend the swap-robustness property and we prove that a necessary and sufficent condition for the extended desirability relation to be complete is that the game (endowed with a coalition structure) be swap-robust. Furthermore for swap-robust $S G C S$, the desirability relation is a complete preorder. Other results of the paper concern the ordinal comparison. It appears that unlike in simple game, $O S$ and $O B$ are not ordinally equivalent even if the game is weighted. Likewise the desirability relation is different from $O S$ and $O B$ in general. However, if structural coalitions have equal size in the partition then both $O B$ and the desirability relation coincide. Also, if $i$ and $j$ are two players belonging to the same structural coalition (they are said to be partners), then $i$ is at least as desirable as $j$ if and only if $i$ has as much power as $j$ according to $O S$.

The remainder of this paper is organized as follows. Section 2 introduces basic notations and definitions. The extension of the desirability relation to 
simple games with coalition structure and some properties are presented in Section 3. Section 4 is devoted to the ordinal comparison of power theories, and Section 5 concludes the paper.

\section{Preliminaries}

\subsection{Simple game}

Throughout the paper $N=\{1,2, \ldots,|N|\}$ is assumed to be the finite set of players. Any non-empty subset of $N$ is called a coalition. A simple game is a couple $(N, W)$, where $W$, the set of winning coalitions on $N$ satisfies monotonicity (i.e. if $S$ is a winning coalition, then any other coalition $T$ containing $S$ is also a winning one). With a simple game $(N, W)$, we associate the mapping $v$ defined by $v(S)=1$ if $S$ is a winning coalition, while $v(S)=0$ if $S$ is not. A weighted voting game is denoted $\left[q ; w_{1}, w_{2}, . ., w_{n}\right]$, where $w_{i} \in \mathbb{N}$ is the number of votes of player $i \in N$, and the quota $q \in \mathbb{N}$ is the minimum number of votes needed to win : $S \in W \Leftrightarrow \sum_{i \in S} w_{i} \geq q$.

In the following, for simplicity $S \cup\{i\}$ will be denoted $S+i$ while $S-i$ represents $S \backslash\{i\}$.

\section{Shapley and Banzhaf indices}

Let $m_{S}^{i}(N, W)=v(S)-v(S-i)$ be the marginal contribution of player $i \in N$ to coalition $S \subseteq N^{1}$ in game $(N, W)$. The well-known Shapley-Shubik index $\left(\varphi^{S}\right)$ and Banzhaf-Coleman index $\left(\varphi^{B}\right)$ are the functions defined by

$$
\begin{gathered}
\varphi_{i}^{S}(N, W)=\sum_{\substack{S \subseteq N \\
i \in S}} \frac{(|S|-1) !(|N|-|S|) !}{|N| !} m_{S}^{i}(N, W) \text { and } \\
\varphi_{i}^{B}(N, W)=\sum_{\substack{S \subseteq N \\
i \in S}} \frac{1}{2^{|N|-1}} m_{S}^{i}(N, W), i \in N .
\end{gathered}
$$

Both of them measure the relative frequency with which a player is in the position of "swinging" a losing coalition into a winning one. We call such a voter a critical defector. A voter $i$ is a critical defector in a coalition $S$ if $S$ is winning while $S-i$ is not $\left(m_{S}^{i}(N, W)=1\right)$.

\footnotetext{
${ }^{1} \subseteq$ denotes inclusion while $\subset$ is the strict inclusion, that is, $A \subset B$ means that $A \subseteq B$ and $A \neq B$.
} 
With every real-valued function $u$ defined on $N$, we can associate a complete preordering on $N$ denoted $\geq_{u}$ and defined by: $i \geq_{u} j \Longleftrightarrow u(i) \geq u(j)$. We denote respectively by $\geq_{S}$ and $\geq_{B}$ the preordering associated with the Shapley-Shubik and Banzhaf-Coleman indices.

\section{Desirability relation}

Introduced by Allingham (1975), the desirability relation is defined as follows:

Definition. Let $(N, W)$ be a simple game, and $i$ and $j$ two players.

i) $i$ and $j$ are said to be equally desirable, denoted by $i \sim_{T} j$ if : for any coalition $S$ such that $i \notin S$ and $j \notin S, S+i \in W \Longleftrightarrow S+j \in W$.

ii) $i$ is said to be more desirable than $j$, denoted by $i>_{T} j$ if the following two conditions are fulfilled:

1) For every coalition $S$ such that $i \notin S$ and $j \notin S, S+j \in W \Rightarrow S+i \in W$

2) There exists a coalition $T$ such that $i \notin T$ and $j \notin T, T+i \in W$ and $T+j \notin W$

The desirability relation (or the influential relation) denoted by $\geq_{T}$ is defined on $N$ as follows: $i \geq_{T} j$ if $i>_{T} j$ or $i \sim_{T} j$. If $i \geq_{T} j$, we say that $i$ is at least as desirable as $j$.

\subsection{Coalition structure}

A coalition structure on $N$ is a finite partition $\mathcal{P}=\left\{P_{1}, \ldots, P_{m}\right\}$ of $m$ nonempty and disjoint subsets of $N$, i.e. $\cup_{k=1}^{m} P_{k}=N$ and $P_{k} \cap P_{l}=\varnothing$ for all $k, l \in\{1, \ldots, m\}, k \neq l$. Note that a coalition structure is assumed to be given exogenously. In the following, an element $P_{k}$ of the partition $\mathcal{P}$ is called a structural coalition (Hamiache 1999), and $n_{k}$ is the cardinality of $P_{k}$. The set of structural coalitions in the coalition structure is denoted by $M=\{1, \ldots, m\}$, with $k \in M$ representing the structural coalition $P_{k} \in \mathcal{P}$. For all $i \in N$, let $k_{i} \in M$ be the index of the structural coalition such that $i \in P_{k_{i}}$. If $k_{i}=k_{j}$ then $i$ and $j$ are said to be partners in $\mathcal{P}$.

A simple game with coalition structure is a triple $(N, W, \mathcal{P})$. Note that there are two trivial partitions: $\mathcal{P}^{N}=\{N\}$ and $\mathcal{P}^{0}=\left\{\{i\}_{i \in N}\right\}$.

A coalition structure index assigns a voting power to any player in ev-

ery simple game with coalition structure $(N, W, \mathcal{P})$. Owen introduced two indices to reflect the outcome of such a game. The first one, introduced in Owen (1977) is a generalization of the Shapley-Shubik index. This index, 
hereafter called the Owen-Shapley index $(O S)$, denoted by $\theta^{O S}$ is defined by

$\theta_{i}^{O S}(N, W, \mathcal{P})=\sum_{L \subseteq M-k_{i}} \sum_{S \subseteq P_{k_{i}}: i \in S} \frac{|L| !(m-|L|-1) !}{m !} \frac{(|S|-1) !\left(n_{k_{i}}-|S|\right) !}{n_{k_{i}} !}[v(S \cup P(L))-v((S-i) \cup P(L))]$ with $P(L)=\bigcup_{t \in L} P_{t}$.

Clearly, this index reduces to $S S$ index when $\mathcal{P}=\mathcal{P}^{N}$ or when $\mathcal{P}=\mathcal{P}^{0}$. The weights of the marginal values are a product of two "Shapley weights", reflecting the fact that first structural coalitions enter subsequently in a random order and that within each structural coalition the players enter subsequently in a random order.

Analogously to the generalization of the $S S$ index, the $B C$ index has been generalized to $S G C S$ in Owen (1981) by replacing in $O S$ the two-level $S S$ weights by the corresponding two-level $B C$ weights. In $B C$ index each marginal contribution has an equal weight. So, generalizing this to $S G C S$ by assigning equal weights to each marginal contribution of a structural coalition within the coalition structure and assigning equal weights to each marginal contribution of the players within that structural coalition, we obtain the generalized Banzhaf index. It is called the Owen-Banzhaf index $(O B)$ and is given by

$$
\theta_{i}^{O B}(N, W, \mathcal{P})=\sum_{L \subseteq M-k_{i}} \sum_{S \subseteq P_{k_{i}}: i \in S} 2^{-(m-1)} 2^{-\left(n_{k_{i}}-1\right)}[v(S \cup P(L))-v((S-i) \cup P(L))] .
$$

We denote by $\geq_{O S}$ and $\geq_{O B}$ the preordering associated with $O S$ and $O B$ respectively.

\section{The desirability relation for simple games with coalition structure}

To write the desirability relation for $S G C S$, we need some additional notation and definitions.

Definition 1. Let $(N, W, \mathcal{P})$ be a $S G C S$, a coalition $S$ is said to be feasible if for all $i, j \in S$ such that $k_{i} \neq k_{j}$, either $P_{k_{i}} \subseteq S$ or $P_{k_{j}} \subseteq S$.

If $S$ is a feasible coalition then $S$ contains the whole structural coalition $P_{k_{i}}$ whenever it contains the player $i$, unless $P_{k_{i}}$ is the unique structural coalition for which $P_{k_{i}} \subset S$.

It can easily be checked that a coalition $S$ is feasible if and only if there exists $L \subset M$ such that $S=P(L) \cup C$, where $P(L)=\bigcup_{t \in L} P_{t}$ and $C \subseteq P_{u}$ with $u \notin L$. 
Definition 2. Let $(N, W, \mathcal{P})$ be a $S G C S, L \subset M$ and $C \subseteq P_{k}$ with $k \notin L$ : a player $i$ is said to be $(L, C)$-critical if $i \in C, S=P(L) \cup C \in W$ and $S-i \notin W$.

Note that this definition generalizes to $S G C S$ the notion of critical defector. We denote by $\mathcal{C}_{i}$ the set of all $(L, C)$ such that $i$ is $(L, C)$-critical; $\mathcal{C}_{i}\left(L, P_{k}\right)$ the set of all coalitions $C \subseteq P_{k}$ such that $i$ is $(L, C)$-critical; $\mathcal{C}_{i}\left(L, P_{k}, c\right)$ the set of all coalitions of $\mathcal{C}_{i}\left(L, P_{k}\right)$ of size $c$.

Definition 3. Let $(N, W, \mathcal{P})$ be a $S G C S, i$ and $j$ two players; $i$ is at least as desirable as $j$ denoted $i \succeq_{O T} j$ if one of the following conditions is fullfilled:

i) either $k_{i}=k_{j}=k$ and for all feasible coalition $S=P(L) \cup C$ with $C \subseteq P_{k}$ such that $i, j \notin C, S+j \in W$ implies $S+i \in W$

ii) or $k_{i} \neq k_{j}$ and $\left|\mathcal{C}_{i}\right| \geq\left|\mathcal{C}_{j}\right|$.

It is clear that if $\mathcal{P}=\mathcal{P}^{N}$ or $\mathcal{P}=\mathcal{P}^{0}$, then the relation defined above coincides with the classical desirability relation so far defined on simple games.

It is also obvious that for some $(N, W, \mathcal{P}), \succeq_{O T}$ may not be complete, that is, there may exists two players $i$ and $j$ such that neither $i \succeq_{O T} j$ nor $j \succeq_{O T} i$. This phenomenon is well-known in simple game without coalition structure. Furthermore, a necessary and sufficient condition under which the desirability relation for simple game is complete is that the game be swap-robust, a concept introduced by Taylor (1995) and recalled below.

Definition 4. A simple game $(N, W)$ is swap-robust if for all $i, j \in N$, all $S, T \in W: i \in S \backslash T$ and $j \in T \backslash S$, at least one of the two coalitions $(S-i)+j$ and $(T-j)+i$ is winning.

Below, we provide a generalization of the swap-robustness property .

Definition 5. A $S G C S(N, W, \mathcal{P})$ is said to be $\mathcal{P}$-swap-robust if : for all feasible winning coalitions $S$ and $T$, all partners $i$ and $j$ such that $i \in S \backslash T$ and $j \in T \backslash S$, either $(S-i)+j \in W$ or $(T-j)+i \in W$.

It is straighforward that the class of $\mathcal{P}$-swap robust games includes the class of weighted $S G C$. $\mathcal{P}$-swap-robustness is a necessary and sufficient condition for the completeness of the desirability relation for $S G C S$ as shown below. 
Proposition 1. Let $R=(N, W, \mathcal{P})$ be a SGCS. The desirability relation $\succeq_{O T}$ is complete if and only if $R$ is $\mathcal{P}$-swap-robust.

Proof. Let $R=(N, W, \mathcal{P})$ be a $S G C S$.

First, assume that $R$ is $\mathcal{P}$-swap-robust. Assume further that $\succeq_{O T}$ is not complete : then there exists $i$ and $j$ such that non $\left(i \succeq_{O T} j\right)$ and non $\left(j \succeq_{O T} i\right)$. Therefore $i$ and $j$ should be partners in $\mathcal{P}$ because if not, either $\left|\mathcal{C}_{i}\right| \geq\left|\mathcal{C}_{j}\right|$ or $\left|\mathcal{C}_{j}\right| \geq\left|\mathcal{C}_{i}\right|$, which is not the case.

Now, $i$ and $j$ are partners in $\mathcal{P}$ and non $\left(i \succeq_{O T} j\right)$ implies that there exist a feasible coalition $S=P\left(L_{1}\right) \cup C_{1}$ with $C_{1} \subseteq P_{k}$ such that $i, j \notin C_{1}$, $S+j \in W$ and $S+i \notin W$.

Likewise, $\operatorname{non}\left(j \succeq_{O T} i\right)$ implies the existence of a feasible coalition $T=$ $P\left(L_{2}\right) \cup C_{2}$ with $C_{2} \subseteq P_{k}$ such that $i, j \notin C_{2}, T+i \in W$ and $T+j \notin W$.

Let $T_{1}=T+i$ and $S_{1}=S+j$. Then, $T_{1}$ and $S_{1}$ are feasible winning coalitions with $i \in T_{1} \backslash S_{1}$ and $j \in S_{1} \backslash T_{1}$. However, both $\left(T_{1}-i\right)+j$ and $\left(S_{1}-j\right)+i$ are losing coalitions and this is a contradiction because the game is $\mathcal{P}$-swap-robust.

Conversely, assume that $R$ is not $\mathcal{P}$-swap-robust : then there exists feasible winning coalitions $S$ and $T$, two partners in $\mathcal{P}, i$ and $j$ such that $i \in S \backslash T$, and $j \in T \backslash S$ and neither $(S-i)+j \in W$ nor $(T-j)+i \in W$.

But $(S-i)+j \notin W$ implies non $\left(j \succeq_{O T} i\right)$ and $(T-j)+i \notin W$ implies non $\left(i \succeq_{O T} j\right)$. Hence $i$ and $j$ are not comparable under the relation $\succeq_{O T}$, which is therefore not complete.

We show below that if the game is $\mathcal{P}$-swap-robust, then $\succeq_{O T}$ is a complete preorder that is a transitive and complete relation.

Proposition 2. Let $R=(N, W, \mathcal{P})$ be a $\mathcal{P}$-swap-robust SGCS. Then the desirability relation $\succeq_{O T}$ is a complete preorder.

Proof. Thanks to Proposition 1, if $R$ is $\mathcal{P}$-swap-robust, then $\succeq_{O T}$ is complete. Let us show that $\succeq_{O T}$ is transitive. Let $i, j, l \in N$ such that $i \succeq_{O T} j$ and $j \succeq_{O T} l$. We need to show that $i \succeq_{O T} l$.

1) First case : $i$ and $l$ are partners in $\mathcal{P}$, that is, $k_{i}=k_{l}=k$.

Let $S=P(L) \cup C$ with $C \subseteq P_{k}$ such that $i, l \notin C$ and $S+l \in W$.

There are two sub-cases : $k_{j}=k$ and $k_{j} \neq k$

Subcase $1: k_{j}=k$ :

If $j \in C$ then $j \in S$ and as $i \succeq_{O T} j$, it follows that $(S-j)+i \in W$, which implies that $S+i \in W$. 
If $j \notin C$ then, $S+j \in W$ since $j \succeq_{O T} l$ and thus $S+i \in W$ because $i \succeq_{O T} j$.

Subcase 2: $k_{j} \neq k$ : assume that $S+i \notin W$. Then $l$ is $(L, C)$-critical meanwhile $i$ is not.

Since $i \succeq_{O T} j$ and $j \succeq_{O T} l$, we have $\left|\mathcal{C}_{i}\right| \geq\left|\mathcal{C}_{j}\right| \geq\left|\mathcal{C}_{l}\right|$, thus, $\left|\mathcal{C}_{i}\right| \geq\left|\mathcal{C}_{l}\right|$. It is the case that there exist $L^{\prime} \subset M, T \subseteq P_{k}$ such that $i$ is $\left(L^{\prime}, T\right)$-critical and $l$ is not $\left(L^{\prime}, T\right)$ because if not, we cannot have $\left|\mathcal{C}_{i}\right| \geq\left|\mathcal{C}_{l}\right|$. Hence, by denoting $S^{\prime}=P\left(L^{\prime}\right) \cup T$, we get : $S^{\prime}+i \in W$ and $S^{\prime}+l \notin W$. Since $S+l \in W$ and $S+i \notin W$, we obtain a contradiction since the game is $\mathcal{P}$-swap robust. Finally, we have $S+i \in W$.

2) Second case : $i$ and $l$ are not partners in $\mathcal{P}$, that is, $k_{i} \neq k_{l}$. We need to show that $\left|\mathcal{C}_{i}\right| \geq\left|\mathcal{C}_{l}\right|$. Again we distinguish two subcases.

Subcase 1: $k_{j}=k_{l}:$ In order to show that $\left|\mathcal{C}_{i}\right| \geq\left|\mathcal{C}_{l}\right|$, it suffices to show that $\left|\mathcal{C}_{j}\right| \geq\left|\mathcal{C}_{l}\right|$, since $\left|\mathcal{C}_{i}\right| \geq\left|\mathcal{C}_{j}\right|$.

Let $(L, S)$ be such that $l$ is $(L, S)$-critical. Players $j$ and $l$ are partners in $\mathcal{P}$ and $j \succeq_{O T} l$.

We will show the following :

(1) : If $l \in S$ then $j$ is $(L, S)$-critical and

(2): if $l \notin S$ then $j$ is $(L,(S-l)+j)$-critical.

- Assume that $l \in S$ : If $j$ is not $(L, S)$-critical, then $(P(L) \cup S)-j \in W$. Let $T_{0}=(P(L) \cup S)-\{j, l\}$. We have $T_{0}+l=(P(L) \cup S)-j \in W$ and $T_{0}+j=(P(L) \cup S)-l \notin W$, which is a contradiction since $j \succeq_{O T} l$. We conclude that if $l \in S$ then $j$ is $(L, S)$-critical.

We can show as well (by contradiction as above) that if $l \notin S$ then $j$ is $(L,(S-l)+j)$-critical.

(1) and (2) above imply that the number of $(L, S)$ in which $j$ is critical is greater than or equal to the number of such couples in which $l$ is critical, that is, $\left|\mathcal{C}_{j}\right| \geq\left|\mathcal{C}_{l}\right|$ and we conclude that $\left|\mathcal{C}_{i}\right| \geq\left|\mathcal{C}_{l}\right|$.

Subcase $2: k_{j} \neq k_{l}$

If $k_{j} \neq k_{i}$, then from $i \succeq_{O T} j$ and $j \succeq_{O T} l$, it follows $\left|\mathcal{C}_{i}\right| \geq\left|\mathcal{C}_{j}\right| \geq\left|\mathcal{C}_{l}\right|$.

If $k_{j}=k_{i}$, we can show as in subcase 1 , that in this case $\left|\mathcal{C}_{i}\right| \geq\left|\mathcal{C}_{j}\right|$ which, together with $\left|\mathcal{C}_{j}\right| \geq\left|\mathcal{C}_{l}\right|$ yield $\left|\mathcal{C}_{i}\right| \geq\left|\mathcal{C}_{l}\right|$.

The relation $\succeq_{O T}$ is transitive and the proof is complete. 


\section{Ordinal equivalence}

Diffo Lambo and Moulen (2002) proved for simple games that $\succeq_{S}$, $\succeq_{B}$ and $\succeq_{T}$ coincide if and only if the simple game is swap-robust. Unlike simple game (without structural coalition), the desirability relation for $S G C S, \succeq_{O T}$ is neither ordinally equivalent to $\succeq_{O S}$ nor to equivalent to $\succeq_{O B}$ even if the game is weighted. Likewise $\succeq_{O S}$ and $\succeq_{O B}$ are different as shown in the following example.

Example 1. Consider the following weighted game $R=[10 ; 3,3,3,2,2,2,1]$ with player set $N=\{1,2,3,4,5,6,7\}$. Assume that players are organized into an a priori coalition structure, such that $\mathcal{P}=\{\{1,2,3\},\{4,5,6\},\{7\}\}$. The Owen-Shapley index and the Owen-Banzhaf index are respectively given by $\theta^{O S}(R)=\left(\frac{2}{9}, \frac{2}{9}, \frac{2}{9}, \frac{1}{18}, \frac{1}{18}, \frac{1}{18}, \frac{1}{6}\right)$ and $\theta^{O B}(R)=\left(\frac{1}{4}, \frac{1}{4}, \frac{1}{4}, \frac{1}{16}, \frac{1}{16}, \frac{1}{16}, \frac{1}{4}\right)$. It can be easily checked that $\succeq_{O T}$ is given by: $1 \sim_{O T} 2 \sim_{O T} 3 \succ_{O T} 4 \sim_{O T} 5 \sim_{O T}$ $6>_{O T} 7$. Thus, $\theta_{1}^{O B}(R)=\theta_{7}^{O B}(R)$ while $1 \succ_{O T} 7$ and $\theta_{7}^{O S}(R)>\theta_{6}^{O S}(R)$ while $6 \succ_{O T} 7$.

Moreover $\theta_{3}^{O B}(R)=\theta_{7}^{O B}(R)$ meanwhile $\theta_{3}^{O S}(R)>\theta_{7}^{O S}(R)$.

We show below that the equivalence relation between $\succeq_{O B}$ and $\succeq_{O T}$ holds if all the structural coalitions in the coalition structure have equal size.

Proposition 3. Let $R=(N, W, \mathcal{P})$ be a $\mathcal{P}$-swap-robust SGCS. If structural coalitions in $\mathcal{P}$ have equal size, then the desirability relation $\succeq_{O T}$ and $\succeq_{O B}$ coincide, that is for all $i, j \in N, i \succeq_{O T} j \Leftrightarrow i \succeq_{O B} j$.

Proof. Consider $R$ a $\mathcal{P}$-swap-robust $S G C S$ such that $\left|P_{k}\right|=\left|P_{k^{\prime}}\right|$ for all $P_{k}, P_{k^{\prime}} \in \mathcal{P}$, two players $i$ and $j$ and assume that $i \succeq_{O T} j$.

We proved in Proposition 2 (second case) that $\left|\mathcal{C}_{i}\right| \geq\left|\mathcal{C}_{j}\right|$ whenever $i \succeq$ OT $j$ and this holds whether $i$ and $j$ are partners or not. Thus, assuming that $i \in P_{k_{i}}$ and $j \in P_{k_{j}}$, we have $i \succeq O T j \Rightarrow\left|\mathcal{C}_{i}\right| \geq\left|\mathcal{C}_{j}\right|$. But,

$$
\begin{aligned}
\theta_{i}^{O B} & =\sum_{L \subseteq M-k_{i}} \sum_{S \subseteq P_{k_{i}}: i \in S} 2^{-(m-1)} 2^{-\left(n_{k_{i}}-1\right)}[v(S \cup P(L))-v((S-i) \cup P(L))] \\
& =\sum_{L \subseteq M-k_{i}} \sum_{S \in \mathcal{C}_{i}\left(L, P_{k_{i}}\right)} 2^{-(m-1)} 2^{-\left(n_{k_{i}}-1\right)} \\
& =\sum_{L \subseteq M-k_{i}} 2^{-(m-1)} 2^{-\left(n_{k_{i}}-1\right)}\left|\mathcal{C}_{i}\left(L, P_{k_{i}}\right)\right| \\
& =2^{-(m-1)} 2^{-\left(n_{k_{i}}-1\right)} \sum_{L \subseteq M-k_{i}}\left|\mathcal{C}_{i}\left(L, P_{k_{i}}\right)\right| \\
& =2^{-(m-1)} 2^{-\left(n_{k_{i}}-1\right)}\left|\mathcal{C}_{i}\right|
\end{aligned}
$$

and 


$$
\begin{aligned}
i \succeq_{O T} j \Rightarrow & \left|\mathcal{C}_{i}\right| \geq\left|\mathcal{C}_{j}\right| \\
\Rightarrow & 2^{-(m-1)} 2^{-\left(n_{k_{i}}-1\right)}\left|\mathcal{C}_{i}\right| \geq 2^{-(m-1)} 2^{-\left(n_{k_{i}}-1\right)}\left|\mathcal{C}_{j}\right|=2^{-(m-1)} 2^{-\left(n_{k_{j}}-1\right)}\left|\mathcal{C}_{j}\right| \\
& \left(\text { since }\left|P_{k_{i}}\right|=\left|P_{k_{j}}\right|\right) \\
\Rightarrow & i \succeq_{O B} j
\end{aligned}
$$

Conversely, assume that $\operatorname{non}(i \succeq O T j)$ : Since $R$ is $\mathcal{P}$-swap-robust, it is the case that $\succeq_{O T}$ is complete, thus non $\left(i \succeq_{O T} j\right)$ implies $j \succeq_{O T} i$ and hence $\left|\mathcal{C}_{j}\right| \geq\left|\mathcal{C}_{i}\right|$.

If $i$ and $j$ are not partners in $\mathcal{P}$, then non $(i \succeq$ OT $j)$ implies $\left|\mathcal{C}_{j}\right|>\left|\mathcal{C}_{i}\right|$.

If $i$ and $j$ are partners in $\mathcal{P}$, then there exists a feasible coalition $S=$ $P\left(L_{2}\right) \cup C$ with $C \subseteq P_{k}$ such that $i, j \notin C, S+j \in W$ and $T+i \notin W$. We therefore have $\left|\mathcal{C}_{j}\right|>\left|\mathcal{C}_{i}\right|$. But $\left|\mathcal{C}_{j}\right|>\left|\mathcal{C}_{i}\right| \Rightarrow 2^{-(m-1)} 2^{-\left(n_{k_{j}}-1\right)}\left|\mathcal{C}_{j}\right|>$ $2^{-(m-1)} 2^{-\left(n_{k_{i}}-1\right)}\left|\mathcal{C}_{i}\right|$, that is, $\operatorname{non}(i \succeq O B j)$.

An obvious consequence of the proposition above is the following :

Corollary 1. Let $R=(N, W, \mathcal{P})$ be a $\mathcal{P}$-swap-robust SGCS. If $i$ and $j$ are partners in $\mathcal{P}$ then $i \succeq$ OT $j \Leftrightarrow i \succeq$ $\succeq_{O B} j$.

We also show that whenever $i$ and $j$ are partners in $\mathcal{P}$, the desirability preordering coincides with the preordering induced by $O S$ index.

Proposition 4. Let $R=(N, W, \mathcal{P})$ be a $\mathcal{P}$-swap-robust SGCS. If $i$ and $j$ are partners in $\mathcal{P}$ then $i \succeq$ OT $j \Leftrightarrow i \succeq$ $j$. $j$.

Proof. Let $R=(N, W, \mathcal{P})$ be a $\mathcal{P}$-swap-robust $S G C S, i$ and $j$ partners in $\mathcal{P}$ such that $i \succeq$ OT $j$. Let $k_{j}=k_{i}=k$.

We have :

$$
\begin{aligned}
\theta_{i}^{O S} & =\sum_{L \subseteq M-k} \sum_{S \subseteq P_{k}: i \in S} \frac{|L| !(m-|L|-1) !}{m !} \frac{(|S|-1) !\left(n_{k}-|S|\right) !}{n_{k} !}[v(S \cup P(L))-v((S-i) \cup P(L))] \\
& =\sum_{L \subseteq M-k} \sum_{S \in \mathcal{C}_{i}\left(L, P_{k}\right)} \frac{|L| !(m-|L|-1) !}{m !} \frac{(|S|-1) !\left(n_{k}-|S|\right) !}{n_{k} !} \\
& =\sum_{L \subseteq M-k} \sum_{s=1}^{n_{k}} \frac{|L| !(m-|L|-1) !}{m !} \frac{(s-1) !\left(n_{k}-s\right) !}{n_{k} !}\left|\mathcal{C}_{i}\left(L, P_{k}, s\right)\right| \text { with } s=|S|
\end{aligned}
$$

Now, we show that $i \succeq_{O T} j$ implies that for all $L \subseteq M-k$ and all $S \subseteq P_{k}$, $\left|\mathcal{C}_{i}\left(L, P_{k}, s\right)\right| \geq\left|\mathcal{C}_{j}\left(L, P_{k}, s\right)\right|$. It suffices, for this purpose to remark that: if $i \succeq$ OT $j$ then :

_ for all $(L, S) \in \mathcal{C}_{j}\left(L, P_{k}, s\right),\left(L, S_{i j}\right) \in \mathcal{C}_{i}\left(L, P_{k}, s\right)$, 


$$
\begin{aligned}
& \text { where } S_{i j}= \begin{cases}S & \text { if } i \in S \\
(S-j)+i & \text { if } i \notin S\end{cases} \\
& \text { _ the mapping } \begin{aligned}
& \psi:\left|\mathcal{C}_{j}\left(L, P_{k}, s\right)\right| \longrightarrow\left|\mathcal{C}_{i}\left(L, P_{k}, s\right)\right| \\
&(L, S) \longmapsto \\
&\left(L, S_{i j}\right)
\end{aligned} \text { is well defined and }
\end{aligned}
$$

is one to one.

$$
\begin{aligned}
& \text { Now, } i \succeq_{O T} j \\
& \Rightarrow\left|\mathcal{C}_{i}\left(L, P_{k}, s\right)\right| \geq\left|\mathcal{C}_{j}\left(L, P_{k}, s\right)\right| \text { for all } L \subseteq M-k \text { and all } S \subseteq P_{k} \\
& \Rightarrow \quad \sum_{L \subseteq M-k} \sum_{s=1}^{n_{k}} \frac{|L| !(m-|L|-1) !}{m !} \frac{(s-1) !\left(n_{k}-s\right) !}{n_{k} !}\left|\mathcal{C}_{i}\left(L, P_{k}, s\right)\right| \geq \sum_{L \subseteq M-k} \sum_{s=1}^{n_{k}} \frac{|L| !(m-|L|-1) !}{m !} \frac{(s-1) !\left(n_{k}-s\right) !}{n_{k} !}\left|\mathcal{C}_{j}\left(L, P_{k}, s\right)\right| \\
& \Rightarrow \theta_{i}^{O S}(N, W, \mathcal{P}) \geq \theta_{j}^{O S}(N, W, \mathcal{P}) \\
& \Rightarrow i \succeq O S j
\end{aligned}
$$

Conversely, if non $\left(i \succeq_{O T} j\right)$ then $j \succ_{O T} i$ because the game is $\mathcal{P}$-swaprobust. But $j \succ_{O T} i$ implies $j \succeq_{O T} i$ and there exist $L_{0} \subseteq M-k, S_{0} \subseteq P_{k}$ such that $j$ is $\left(L_{0}, S_{0}\right)$ critical and $i$ is not. Therefore,

$$
\begin{aligned}
& \left\{\begin{array}{l}
\left|\mathcal{C}_{j}\left(L, P_{k}, s\right)\right| \geq\left|\mathcal{C}_{i}\left(L, P_{k}, s\right)\right| \text { for all } L \subseteq M-k \text { and all } S \subseteq P_{k} \quad \text { and } \\
\left|\mathcal{C}_{j}\left(L_{0}, P_{k},\left|S_{0}\right|\right)\right|>\left|\mathcal{C}_{i}\left(L_{0}, P_{k},\left|S_{0}\right|\right)\right|
\end{array}\right. \\
& \text { Hence, } \operatorname{non}(i \succeq O T j) \\
& \Rightarrow \quad \sum_{L \subseteq M-k} \sum_{s=1}^{n_{k}} \frac{|L| !(m-|L|-1) !}{m !} \frac{(s-1) !\left(n_{k}-s\right) !}{n_{k} !}\left|\mathcal{C}_{j}\left(L, P_{k}, s\right)\right|>\sum_{L \subseteq M-k} \sum_{s=1}^{n_{k}} \frac{|L| !(m-|L|-1) !}{m !} \frac{(s-1) !\left(n_{k}-s\right) !}{n_{k} !}\left|\mathcal{C}_{i}\left(L, P_{k}, s\right)\right| \\
& \Rightarrow \quad \theta_{j}^{O S}(N, W, \mathcal{P})>\theta_{i}^{O S}(N, W, \mathcal{P}) \\
& \Rightarrow \quad n o n(i \succeq O S j) \\
& \text { and the proof is complete. }
\end{aligned}
$$

\section{Conclusion}

After extending the desirability relation to $S G C S$, we determine a necessary and sufficient condition for which the desirability relation of a $S G C S$ is a complete preorder. We move on to the question motivating this study, which is whether the preordering induced by the desirability relation of a $S G C S$ coincides with the preorderings induced by the Owen-Shapley and the OwenBanzhaf indices, respectively. The negative answer to this question is one of the main message of our analysis. Indeed, it appears that in general they are different even if one restrict on the class of weighed games. However, if structural coalitions have equal size in the partition then both Owen-Banzhaf and the desirability preordering coincide. Moreover if two players are partners in the same structural coalition, they are compared in the same way under both Owen-Banzhaf preordering and the desirability relation. 
It is worth noting that voting games in his nature does not take into account the possibility for voters to abstain. The extension of the desirability relation to ternary voting game (which take into account abstention) can be found in Pongou et al. (2011). In Tchantcho et al. (2008) the problem of ordinal equivalence is adressed and extended later on by Parker (2012). It may seems appropriate to extend our results to ternary voting games with coalition structure.

\section{References}

[1] Allingham, M.G. (1975). Economic power and values of games. Z. Nationalökon, 35, 293-299.

[2] Andjiga, N., Chantreuil, F., \& Lepelley, D. (2003). La mesure du pouvoir de vote. Mathématiques et sciences humaines, 163, 111-145.

[3] Aumann, R.J., \& Drèze, J.H. (1974). Cooperative games with coalition structures. International Journal of Game Theory, 3, 217-237.

[4] Banzhaf, J. (1965). Weighted voting doesn't work: A mathematical analysis. Rutgers Law Review, 19, 317-343.

[5] Coleman, J.S. (1971). Control of collectivities and the power of a collectivity to act. In B. Lieberman (Ed.), Social Choice (pp. 269-300). New York: Gordon and Breach.

[6] Diffo Lambo, L., \& Moulen, J. (2002). Ordinal equivalence of power notions in voting games. Theory and Decision, 53, 313-325.

[7] Hamiache, G. (1999). A new axiomatization of the Owen value for games with coalition structures. Mathematical Social Sciences, 37, 281-305.

[8] Isbell, J.R. (1958). A class of simple games. Duke Mathematical Journal, 25, 423-439.

[9] Laruelle, A., Valenciano, F. (2008). Voting and Collective DecisionMaking. Cambridge: Cambridge University Press. 
[10] Owen, G. (1977). Values of games with a priori unions. In R. Hernn, O. Moschlin (Eds.), Lecture Notes in Economics and Mathematical Systems: Essays in Honor of Oskar Morgenstern (pp. 76-88). New York: Springer-Verlag.

[11] Owen, G. (1981). Modification of the Banzhaf-Coleman index for games with a priori unions. In M.J. Holler (Ed.), Power, voting and voting power (pp 232-238). Wurzburg: Physica-Verlag.

[12] Parker, C. (2012). The influence relation for ternary voting games. Games and Economic Behavior, 75, 867-881.

[13] Pongou, R., Tchantcho, B., \& Diffo Lambo, L. (2011). Political influence in multi-choice institutions: cyclicity, anonymity and transitivity. Theory and decision, 70, 157-178.

[14] Shapley, L.S., Shubik, M. (1954). A model for evaluating the distribution of power in a committee system. American Political Science Review, 48, $787-792$.

[15] Taylor, A.D. (1995). Mathematics and Politics - Strategy, Voting, Power and Proof. Berlin: Springer-Verlag.

[16] Tchantcho, B., Diffo Lambo, L., Pongou, R., \& Mbama Engoulou, B. (2008). Journal Voters' power in voting games with abstention: Influence relation and ordinal equivalence of power theories. Games and Economic Behavior, 64, 335-350.

[17] Tomiyama, Y., (1987). Simple game, voting representation and ordinal power equivalence. International Journal on Policy and Information, 11, $67-75$. 